\title{
PREFERENCIA ASPECTUAL EN EL LÉXICO Y COMPENSACIÓN MORFO-SINTÁCTICA: ASPECTOS DEL CONTRASTE INGLÉS-ESPAÑOL
}

\author{
ASPECTUAL PREFERENCES IN THE LEXICON AND MORPHO- \\ SYNTACTIC COMPENSATION: THE SPANISH-ENGLISH \\ CONTRAST
}

\author{
LUIS PARÍS \\ INCIHUSA-CONICET; UNCuyo. Mendoza, Argentina \\ paris@mendoza-conicet.gob.ar
}

\section{RESUMEN}

En este trabajo se defienden dos hipótesis complementarias referidas a la construcción de representaciones de eventos. La primera es que el inglés exhibe una propensión a contener semánticas léxicas verbales que correspondan a Actividades mientras el español favorece la codificación léxica de Eventos. Se denomina a esta tesis 'preferencia aspectual'. La segunda hipótesis -se denomina 'compensatoria'- es que las dos lenguas cuentan con construcciones sintácticas que, al introducir el recurso descriptivo menguado -Actividad o Evento, respectivamente-, compensan los desbalances léxicos provocados por sus preferencias aspectuales. La Construcción de Gerundio Interno (CGI) en español permite introducir Actividades que especifican el desarrollo de un Evento. La Construcción Resultativa del inglés permite añadir un Resultado al final de una Actividad trocándola así en un Evento. La interpretación 'compensatoria' es consistente con la carencia de CGI en inglés y de CR en español. Este contraste de esquemas gramaticales para la representación de eventos se refleja también en la diátesis causativa. El punto de partida en inglés es la semántica más simple, mono-eventiva, que se enriquece mediante procedimientos morfosintácticos al incorporar otro un subevento causal. Por el contrario, el español no enriquece sino que sustrae la causalidad de un Evento complejo mediante morfosintaxis.

Palabras clave: Eventos, aspecto léxico, construcción resultativa.

\section{ABSTRACT}

In this paper we propose two complementary hypotheses on the construction of event representations. The first one is that English displays a preference for codifying Processes in the semantic representations of verbs while Spanish favors Events. We call this thesis 'aspectual preference'. The second hypothesis -called 'compensatory'- is that the two 
languages have syntactic constructions that introduce the devalued semantic resource either Process or Event, respectively- and, therefore, these constructions counterweight the unbalance produced by the aspectual preferences in the lexicon. The Spanish Internal Gerund Construction (IGC) allows speakers to introduce Processes that specify the internal parts of an Event. The English Resultative Construction (RC) permits speakers to introduce Results into Processes that, hence, become enclosed and turned into Events. The compensatory interpretation is further backed up by absence of IGC in English and the lack of RG in English. This contrast between the procedures for construction of event representations is further corroborated by the causative alternation. The point of departure in English is a simple, mono-event semantics that gets richer by incorporating a causative subevent through syntactic means. In contrast, the Spanish strategy is not of enrichment but of extraction: A complex Event is split apart by morphosyntax.

Keywords: Events, lexical aspect, resultative construction.

Recibido: 08.04.2014. Aceptado: 20.01.2015.

\section{INTRODUCCIÓN}

$\mathrm{H}$ ablamos mediante oraciones, solo ellas pueden acarrear un mensaje (verdadero o falso). Los verbos son sus núcleos semánticos ya que aportan la red de relaciones que vinculan entre sí a los individuos participantes del evento denotado por la oración. Al interior del significado verbal se distingue un componente estructural y otro de puro contenido - denominado 'raíz' - y que contiene la riqueza informativa de cada verbo, su riqueza conceptual. En el léxico esta representación conceptual es organizada y estructurada por un conjunto muy reducido de esquemas semánticos que resaltan cierta información y soslayan otra gramaticalmente invisible. Todos los verbos de todas las lenguas pertenecen inevitablemente a alguno de los esquemas asociados a las categorías aspectuales de Estado, Actividad y Evento (Vendler, 1957; Van Valin, 2005). Cada una remite a un tipo distinto de desarrollo en el tiempo de la relación entre individuos invocada por el significado de un verbo. Las lenguas tienden a dar un tratamiento análogo a los miembros de una misma categoría aspectual, es decir, existe evidencia morfosintáctica de la visibilidad gramatical de cada una de esas categorías (entre otros, véase Van Valin, 1990; Tenny, 2000).

Los Estados son situaciones que se mantienen de manera homogénea en el eje temporal; es decir, son durativas pero no tienen dinámica y de aquí que, por ejemplo, no pueden combinarse con formas progresivas (*Pedro está sabiendo matemáticas'). Son abiertos, no involucran un límite o meta intrínseca que deba obtenerse para que se cumpla la situación. Las Actividades son también situaciones homogéneas, durativas y abiertas pero, a diferencia de los Estados, son diná- 
micas, siempre incluyen un participante Actor por lo que la oración puede responder '¿Qué hizo ese participante?'. 'Corrió' es una respuesta posible pero no lo es 'supo', ya que 'saber' denota un estado. Las Actividades pueden, en consecuencia, expresarse en aspecto progresivo ('Pedro está/estuvo corriendo en el parque'). Los Eventos, en cambio, no son homogéneos porque involucran en su interior un subevento que es necesariamente diferente al curso de eventos precedente; es decir, codifican un cambio que debe completarse como condición para el uso del verbo. Este cambio refiere a un subevento diferente al final del Evento y por esto se los categoriza como verbos télicos (del griego 'telos' (fin)). Los Eventos pueden ser durativos -involucran un proceso que termina en una Meta- como 'derretir' y se denominan Realizaciones ('Accomplishments') o pueden ser puntuales -como 'explotar' o 'llegar'- y se denominan Logros ('Achievements').

El estudio que se presenta aquí analiza contrastivamente un aspecto fundamental de los estilos en la construcción de representaciones de eventos en inglés y español ${ }^{1}$. La primera hipótesis es que el inglés tiende a seleccionar representaciones semánticas del tipo Actividad como significado de sus verbos, mientras el español prefiere el tipo Evento. El autor denomina a este contraste en la semántica léxica 'preferencia aspectual'. La segunda hipótesis es que el desequilibrio semántico del léxico inducido por esta preferencia aspectual encuentra su balance en la morfosintaxis. En particular, la Construcción Resultativa del inglés introduce Resultados -típicamente mediante una frase adjetiva predicativa- en una oración constituida por un verbo principal que denota una Actividad. En contraste, el español posee la Construcción de Gerundio Interno que permite introducir una Actividad inherente al Evento descripto por el verbo principal. Y denomina a esta segunda hipótesis 'compensación'. Resulta crucial para fundamentar la relación entre ambas hipótesis que, tal como de hecho acontece, el español carezca de la Construcción Resultativa y que el inglés adolezca de la Construcción de Gerundio Interno.

Las representaciones de eventos son tan elementales, recurrentes e imprescindibles para la descripción de nuestra experiencia que una diferencia de estilo en su construcción desparrama sus efectos sobre todo el sistema. Cada lengua late según su propio 'espíritu' al decir de Von Humboldt o, en palabras de Coseriu, tiene 'su propio pensamiento idiomático'. Este estudio se encolumna detrás de las investigaciones que han auscultado la lógica básica con la que una lengua nos

\footnotetext{
${ }^{1}$ La categoría 'Evento' puede inducir a equivocaciones que intento salvar mediante el uso de mayúscula para la categoría aspectual mientras que uso minúsculas ('evento') para referir a cualquier tipo de entidad temporal denotada por un verbo cualquiera y que, por lo tanto, podría incluir alternativamente a cada una de las categorías aspectuales. Se trata de un problema terminológico inherente a la literatura semántica que en inglés se intenta salvar recurriendo a 'eventuality' para 'evento' en minúsculas, pero cuya traducción al español ('eventualidad') tiene un significado muy distinto que haría su uso aquí problemático.
} 
permite hablar del mundo y de nosotros mismos y que, explícita o o implícitamente, favorecen la perspectiva del relativismo lingüístico. Si bien la evidencia para sostener un relativisimo whorfiano debería considerar el impacto cognitivo de las estructuras lingüísticas -y este punto no es tematizado aquí-, numerosos estudios han mostrado ya que los esquemas cognitivos de los hablantes están condicionados por los recursos que la lengua pone a su disposición (Bowerman y Choi, 2003; Gentner y Bowerman, 2009; Gennari, Sloman, Malt y Fitch, 2002; como así también los trabajos de Slobin $(2000,2006)$ respecto de la tipología de Talmy (2000) en particular).

Esta investigación es contrastiva y teórico-descriptiva. Es contrastiva porque se comparan esquemas gramaticales de dos lenguas entre sí en relación a un mismo dominio cognitivo, que sirve de tertium comparationis. Se trata de ver qué diferencias existen entre los recursos que ponen a disposición dos lenguas para reportar un mismo segmento de realidad -por ejemplo, el movimiento o el cambio de estado físico o una misma relación de interacción entre las mismas entidades. Es descriptiva porque dedica un espacio significativo a la descripción de datos de las dos lenguas. Finalmente, tiene una base teórica al enunciar hipótesis que proponen patrones generales y abstractos para explicar un contraste entre dos lenguas. Además, las hipótesis se asientan en categorías de aspecto léxico que, propuestas como universales semánticos, forman parte en uno u otro modo de posiblemente todas las teorías gramaticales predominantes en la comunidad lingüística (Minimalism, HPSG; Role and Reference, Lexical Functional Grammar, entre otras). Si bien cada una de las categorías semánticas usadas aquí recibe una representación formal en la literatura semántica, he obviado apelar a formalismos porque, si bien añadirían precisión y claridad, no harían ninguna contribución conceptual significativa a la propuesta y, al mismo tiempo, introducirían tecnicismos que, o bien son explicados restando así espacio para contenido, o bien representarían un obstáculo para la lectura del artículo.

El plan de este trabajo es el siguiente. En la segunda sección expongo sucintamente la propuesta de Talmy (2000) que sirve como base de la hipótesis de la preferencia y muestro cómo ésta se desarrolla a partir de aquélla gracias a una generalización sobre las nociones de Manera y Resultado. En la tercera sección presento la hipótesis de la compensación. En la cuarta muestro el éxito limitado de cualquier intento de traducción de una lengua a la otra en relación con la Construcción Resultativa y la Construcción de Gerundio Interno. La quinta sección considera la similitud entre los procesos de ensamblaje gramatical de cada una de las lenguas en relación a las estructuras compensatorias ya descritas y otros ensamblajes gramaticales referidos a distintas representaciones de eventos. Finalmente, en la conclusión expongo sumariamente los resultados de las secciones anteriores y las investigaciones que quedan por hacer para fundamentar más aun la preferencia aspectual. 


\section{LA PREFERENCIA ASPECTUAL COMO GENERALIZACIÓN DE LA TIPOLOGÍA DE TALMY}

Talmy $(2000,2009)$ muestra que el inglés representa un evento de movimiento ('Motion Event') expresando la Manera (walk 'caminar') en el verbo principal y su Meta en un satélite preposicional (into 'hasta-y-dentro'). En contraste, el español capta la misma situación codificando el cambio de Lugar -y por lo tanto, la Meta- en el verbo principal mientras obvia Manera. Las oraciones (1) y (2) son los equivalentes coloquiales más cercanos entre las dos lenguas.

(1) The kid walked into his bedroom.

(2) El niño entró a su dormitorio.

La situación en el mundo es la misma y, potencialmente, también lo son los componentes cognitivos del movimiento: el participante que se mueve (Figura o Tema), un Trayecto con un Origen y una Meta como límites y una Manera de recorrerlo, un cambio de Lugar (de algún modo superpuesto al Tema y a los componentes del Trayecto) y, finalmente, una relación de Movimiento que vincula todos esos componentes en una unidad. La expresión lingüística solo codifica alguno de esos componentes, selección que puede variar de una lengua a la otra, como lo muestra el contraste español-inglés. La semántica léxica del verbo principal de la oración inglesa (2) contiene Tema, Trayecto abierto (esto es, sin Origen ni Meta) y Manera de movimiento; la Meta del Trayecto es introducida mediante un constituyente ajeno al verbo ('into-NP'). En contraste, el verbo principal en español codifica Tema y cambio de Lugar. Trayecto y Manera de movimiento son, por el contrario, soslayados. Meta es el cierre o límite que enmarca al evento motriz; de ahí que, dado que en inglés aparece en el satélite 'into', Talmy clasifique a la lengua como 'satellite framed. El español es 'verb framed' ya que ese límite (Meta) del evento es introducido por el significado del verbo principal. El verbo walk-tanto como 'caminar' - es una Actividad, es decir, una situación dinámica, durativa y abierta; su significado, entonces, no incluye una Meta que deba ser alcanzada. Más específicamente, es un verbo de Manera de Movimiento a través de un Trayecto. El verbo 'entrar', en cambio, no codifica un Trayecto ya que, como se muestra en París $(2010,2011)$, no puede predicarse de ese supuesto Trayecto una frase de medida ('\#Pedro entró a la cocina dos metros') y no es durativo ('Pedro entró a la cocina en cinco minutos' no entraña 'estuvo entrando durante cinco minutos'). 'Entrar' refiere a un Evento temporalmente puntual, es un Logro. ¿De qué Manera cambió el Tema de Lugar? La oración (1) no especifica ese tipo información a través de una forma explícita; sin embargo, ella está conceptualmente presente, es decir, si un participante Tema entró (a algún lugar), recorrió un Trayecto. No 
concebimos un movimiento donde un participante Tema 'salte' un espacio sin pasar por sus puntos intermedios; existe un Trayecto que fue, además, recorrido de alguna Manera. El español no especifica pero sí comunica esa información (París, 2008). En una situación como la descripta en (2), dado el tipo de Trayecto y su dimensión y dado el tipo de Tema (un humano), recorrerlo caminando es lo necesariamente esperable y, por lo tanto, se lo infiere mediante una implicatura conversacional generalizada a partir del Principio de Minimización (Levinson, 2000). De hecho, expresar Manera de movimiento -ejemplo (3) - es inapropiado en un contexto no marcado. El español obliga a obviar Manera si puede inferirse (o es irrelevante) ya que la frase de gerundio 'caminando' atrae el rasgo Foco y, por lo tanto, presenta esa información como la más saliente (París, 2006a). En (3) 'caminando' solo podría ser saliente en un contexto muy marcado, uno donde fuera totalmente sorpresivo que ese niño caminara.

(3) \#El niño entró a su dormitorio caminando.

La tipología de Talmy puede ser reinterpretada y generalizada. En Rappaport y Levin (2010) se propone y se defiende convincentemente que las lenguas manifiestan una tendencia universal a evitar la codificación conjunta de información específica sobre Manera y Resultado en la semántica de un mismo verbo (véase también Beaver y Koontz-Garboden, 2012). Si un verbo describe en su semántica Manera no puede también especificar el Resultado -que, en el domino del movimiento es Meta- y viceversa. En consecuencia, si la oración se estructura a partir de Manera, el Resultado solo puede aparecer como adjunto. Del mismo modo, si el núcleo verbal introduce Resultado, la Manera solo puede ser un adjunto. Esta generalización es consistente con la tipología de Talmy².

Se propone aquí que es posible dar un paso más en el nivel de generalización al subsumir la noción de Resultado en la de Evento y la de Manera en la de Actividad. Toda semántica léxica que codifique un Resultado necesariamente pertenece a la categoría aspectual Evento. Por definición, Resultado remite a un cambio producido al final de un curso de eventos, es decir, se trata de una situación con un fin intrínseco, un curso de eventos 'heterogéneo' que activa la noción de 'completud': solo una vez alcanzado ese fin puede decirse que la situación se completó. Se asume aquí, además, que la relación Manera entre (dos descripciones de) eventos involucra una Actividad como 'Manera de' el otro evento ${ }^{3}$. Es decir, el evento que funcio-

\footnotetext{
${ }^{2}$ Existen ejemplos que contradicen esa tesis. Goldberg (2010) refiere los verbos franceses 'sauté' o 'braise' que codifican al mismo tiempo Manera y Resultado. Beaver y Koontz-Garboden (2012) mencionan los verbos de Manera de matar ('degollar', 'envenenar', 'guillotinar', 'acuchillar', etc.). Lo relevante aquí es que, aun con contraejemplos, esa generalización capta una tendencia significativa y visible.

3 'Manera' es aquí una relación entre situaciones tal que una situación es 'Manera de' otra situa-
} 
na como 'Manera de' es dinámico y, por lo tanto, involucra un participante Actor.

La generalización que resulta de la reformulación de la tipología de Talmy es la siguiente. El léxico inglés privilegia la especificación de Actividades mientras que el léxico del español tiende a describir Eventos. No se afirma que el español no codifique Actividades o que el inglés no codifique Eventos, sino que lo hacen, respectivamente, pero en un menor número relativo. Existe, en consecuencia, una 'preferencia aspectual' contrastante en ambas lenguas.

La validación de la 'preferencia aspectual' la encontramos y de manera fundamental en el estudio contrastivo a partir de la hipótesis de la 'compensación': ambas lenguas equilibran sus respectivas tendencias léxicas mediante combinaciones morfosintácticas que expresan la información soslayada en el verbo principal.

\section{LA COMPENSACIÓN}

El español compensa su austeridad léxica respecto de Actividades con una construcción especializada en la expresión de los mismos: la construcción adverbial de gerundio interno (CGI) representada en los ejemplos en (4).

(4) a. Pedro construyó el puente juntando troncos.

b. Pedro llegó sonriendo.

En (4a) 'construir' refiere a un cambio de estado de un Paciente (el puente) causalmente provocado por un Agente (Pedro). Los verbos causativos típicamente no detallan el evento causante y el gerundio auxilia esa información. En ambos casos la frase de gerundio expresa el Medio por el cual se llevó a cabo el evento principal: los dos eventos están en el mismo curso de eventos (París, 2006b). En cambio, en (4b) el evento principal 'llegar' y el evento gerundio 'sonreír' están en diferentes cursos de eventos, mantienen una relación 'incidental' (París, 2006c) y pueden eventualmente ser expresados por construcciones adverbiales con un orden invertido ('Pedro sonreía al llegar'). En todos los casos el evento gerundio es interpretado como parte propia del evento principal, para lo cual resulta crucial el aspecto 'imperfectivo' del gerundio que focaliza los estadios intermedios de desarrollo del evento mientras deja sin especificar sus límites (inicial o final). Esto lo vuelve particularmente apto para ser introducido como parte de otro evento.

ción tal que en 'el niño entró a su habitación gateando', gatear es la manear de entrar. En particular, se trata aquí de la relación Medio (París, 2006b) ilustrada en casos como (2) arriba y (4a) abajo. El otro tipo de Manera es Incidental y en este caso los eventos no guardan entre sí una relación de tipo causal (por ej. 'entrar llorando'). En cualquier caso, el sentido que uso aquí es más restrictivo que el uso de 'manera' como un tipo de modificación adverbial. 
Se trata, entonces, de un único evento (por ej. 'entrar gateando') y esta unidad se manifiesta en la sintaxis. El gerundio -que es aquí un adjunto, es decir, un elemento sintáctica y semánticamente opcional-, opera en esta construcción como un complemento sintáctico (París y Koenig, 2003).

$\mathrm{El}$ inglés carece de esta construcción casi por completo. La forma ' $\mathrm{V}+$ +ing' que se relaciona con nuestro gerundio toma distintos roles, es una 'categoría mixta' (Malouf, 2000). Una de sus funciones es nominal, es la forma típica que toma el verbo en posición de sujeto ('Smoking is not healthy', "Fumar no es saludable"). Además, es obligatorio luego de una preposición ('John broke the door by smashing it hard with a bat', "John rompió la puerta pegándole fuerte con un palo"). Estos usos son, a diferencia del gerundio español, aspectualmente perfectivos. Existe además un uso adjetival muy frecuente del gerundio como en 'The developing world is at stake here' ("El tema aquí es el mundo en vías de desarrollo/ que se desarrolla”), función que en español no es posible ${ }^{4}$. Por otro lado, y lo que es más importante, el uso verbal del gerundio en una frase con función adverbial -como en español CGI- está severamente restringido. Por un lado, no es posible un gerundio verbal que involucre la relación Medio como en (5a) y (5b).

a. \#John arrived running.

'John llegó corriendo'.

b. \# John bought the house saving dollars.

'John compró la casa ahorrando dólares'

Tampoco es posible de manera productiva generar oraciones con gerundio verbal que codifiquen la relación Incidental como se ve en (6).

(6) Juan cocinó el pollo mirando televisión.

'\#Juan cooked the chicken watching TV

En contraste, sí es posible el gerundio adverbial con una relación Incidental si el verbo principal es un Evento télico y de movimiento, como lo muestra (7).

(7) John came home singing. (ejemplo de Truswell, 2007).

'Juan vino a casa cantando'

París (2013) propone que la combinación 'verbo de movimiento télico+gerundio' instancia un esquema semántico que recibe un trato especial por parte de la gra-

\footnotetext{
${ }^{4}$ Esa es la visión tradicional y es también la de la última gramática de la RAE (2011). Si bien es discutible, es evidente que de ser posible, el gerundio adjetival no es productivo.
} 
mática tanto en español como en muchas otras lenguas. Esta condición especial es consistente con la propuesta en Foley y Olson (1985), según la cual los verbos télicos de movimiento son necesariamente parte de cualquier lengua que tenga construcciones seriales. El inglés permitiría esta construcción -a la que subyace una ligazón sintáctica cercana a una estructura serial- solo con estos verbos debido a características semánticas específicas (télicos y de movimiento).

En contraste, el inglés necesita balancear su preferencia léxica con una sintaxis que permita expresar la culminación de una Actividad en un Resultado. Esta es precisamente la función de la Construcción Resultativa (CR), construcción ejemplificada en (8) (Rothstein, 2000; Goldberg y Jackendoff, 2004; Rappaport y Levin, 2010).

(8) The critics laughed the play off the stage.

Los críticos rieron de obra fuera el escenario

'Los críticos sacaron la obra de cartelera riéndose de ella'

El verbo principal laugh ("reír") es intransitivo y denota una Actividad. Sin embargo, su combinación sintáctica con un satélite ('off') en el contexto de la Construcción Resultativa permite la expresión de un resultado final en la forma de un cambio de Lugar. La interpretación es clara y unívoca: la Actividad fue la causa del estado final que, así, se convierte en Resultado. La relación semántica causal, ausente en el verbo principal y en el satélite, es introducida por la construcción misma.

El motor de generación semántica del inglés consiste en tomar una Actividad léxica como centro y añadir sintácticamente material en la periferia que la haga variar en distintos sentidos. Analicemos, por ejemplo, el verbo sweep (“barrer"). Tanto este verbo inglés como el 'barrer' español denotan una Actividad física que involucra cuatro participantes, una Manera de ejecutar la Acción del Actor y una relación causal. Los participantes son: un Actor que ejecuta la Actividad, un Instrumento, un Tema que refiere a lo removido (tierra, suciedad, polvo, etc.) y un objeto Lugar (una superficie como un piso, una mesa, un escritorio, el techo de un auto, etc.) de donde se mueve el Tema. La Manera refiere a una acción iterativa del Actor con un Instrumento que entra en contacto con distintas partes del Lugar. La relación causal se da entre la acción del Actor y el cambio de Lugar del Tema. Tanto sweep como 'barrer' exhiben realizaciones alternativas en sus usos transitivos. El participante afectado y, por lo tanto realizado como objeto directo, puede ser tanto el Tema como el Lugar, como ejemplifico en $(9 \mathrm{a} / \mathrm{b})$ y $(10 \mathrm{a} / \mathrm{b})$, respectivamente.

(9) a. Terry swept the floor.

b. Each should sweep the leaves from before his own front door. 
a. Pedro barrió el piso.

b. Cada uno debe barrer las hojas de su propia entrada.

Esta realización alternativa también se constata en verbos como load y 'cargar' y, después de Dowty (1991), existe consenso sobre un correlato semántico de la diferente realización sintáctica. Si el Tema es expresado como objeto directo -como en (9b) y (10b) - no se entraña que el Lugar fue totalmente afectado. En cambio, si el Lugar funciona como objeto directo tal como en (9a) y (10a), se torna Incremental, es decir, se entraña que todo el piso fue barrido y, entonces, totalmente afectado. (9a) o (10a) serían falsas si solo se hubiese barrido una pequeña parte del piso $^{5}$. El inglés toma la semántica básica del verbo sweep y la completa con diversos sentidos.

a. Terry swept.

'Terry barrió'

b. Terry swept the floor.

'Terry barrió el piso’

c. Terry swept the leaves.

'Terry barrio las hojas'

d. Terry swept the leaves into the corner.

'Terry puso las hojas en un rincón barriéndolas/al barrerlas'

e. Terry swept the leaves off the sidewalk.

'Terry barrió las hojas de la vereda'

f. Terry swept the floor clean.

\footnotetext{
5 Sweep denota, además, una Manera más genérica -es decir, menos específica- que admite distintos tipos de instrumentos mientras que el español remite solo a una escoba o cepillo y el Lugar es típicamente algún tipo de piso, es decir, una superficie para pisar. El inglés centra su semántica en el tipo de 'movimiento con contacto' del Tema con respecto a una superficie. 'Sweep a brush over the canvas' or 'Sweep the chimney with a brush' no pueden traducirse con nuestro 'barrer'.
} 
Preferencia aspectual en el léxico y compensación morfo-sintáctica: Aspectos del contraste inglés-español / L. PARís

'Terry limpió el piso barriéndolo'

g. Terry swept the leaves into a pile.

'Terry juntó las hojas en una pila barriéndolas/al barrerlas'

En (11a) está usado intransitivamente con un objeto directo inespecífico, es decir, se asume que se barrió algo. En (11b) el objeto directo es el Lugar/Incremental, un objeto afectado 'débilmente' ya que el verbo no entraña un cambio de estado (no necesariamente el piso está limpio luego de barrerlo), aunque se lo implica. En (11c) el objeto directo, en cambio, no es el Tema, la sustancia removida y de la que solo se predica un movimiento. Hasta aquí el español puede ofrecer un reflejo de cada ejemplo inglés; a partir de (11d) comienzan los desacoples. El inglés permite expresar la Meta donde termina localizado el Tema mediante un constituyente interno a la cláusula de la que el verbo de Manera es el núcleo; esta localización se interpreta como el Resultado del evento principal. Para construir una representación de evento cercana a la del inglés, el español debe recurrir a un verbo causativo de cambio de posición ('poner') y expresar la Manera en un gerundio u otro tipo de adjunto. En (11e) es el Origen del Trayecto -y no la Meta- lo que está integrado a la cláusula principal. La expresión más cercana de esa información permite captar al Origen de manera inferencial al codificarlo al interior de la frase nominal en posición de objeto directo ('las hojas de la vereda'). El ejemplo (11f) es una instanciación típica de la Construcción Resultativa. Una frase adjetiva ('clean') describe la propiedad que el Lugar Incremental obtiene como resultado de la Actividad descrita por el verbo. Este constructo no puede ser replicado en español (“\#Terry barrió el piso limpio’). Finalmente, (11g) es también una Construcción Resultativa donde el Resultado es captado por una frase preposicional que introduce la constitución de una entidad (una pila de hojas) como cierre el evento.

El análisis de otro tipo semántico de Actividad debería resaltar aún más el patrón subyacente a la construcción de eventos en una y otra lengua. Este es el caso de 'whistle' ("silbar"), un verbo que codifica una Actividad de producir de sonidos. Se trata de un verbo intransitivo -(12a)- que involucra un Actor humano que produce un sonido lexicalizado en el verbo de una Manera particular: exhalando aire por la boca con los labios formando un círculo. En (12b) se convierte en un verbo de comunicación al incorporar un receptor mientras que (12c) es una versión transitiva con un sonido específico -con una estructura interna- como objeto directo. El ejemplo (12d) es particularmente interesante porque, primero, refleja un patrón muy productivo en inglés y, segundo, no es posible reflejarlo en español. Si bien el verbo codifica un sonido como objeto directo, en este ejemplo aparece como tal una entidad que no se reduce a un sonido. Una advertencia es 
un mensaje con un contenido específico, y, por lo tanto, el medio de transmisión (sonido, grafía, señales visuales, etc.) no es el centro de su significado. Sin embargo, 'advertencia' puede ser objeto directo de whistle con la interpretación 'producir una advertencia mediante/como resultado de un silbido'. El mismo esquema interpretativo subyace a un ejemplo como 'Kim whistled her appreciation' ("Kim expresó su aprecio mediante un silbido/silbando") donde 'aprecio' tampoco es una realidad que tenga una conexión intrínseca con un sonido. El ejemplo (12e) muestra que en las representaciones precedentes se puede incluir además al participante Receptor que funcionaría como parte del Resultado al ser la Meta donde termina localizado el mensaje. Estos últimos tres ejemplos son particularmente ilustrativos de la plasticidad del inglés para construir representaciones de eventos con Actividades, plasticidad que refiere precisamente al vínculo causal entre la acción ejecutada por el Actor -la producción de un sonido de una determinada Manera- y el producto. Más allá de una licencia poética, el español solo permite un Paciente -es decir, el objeto creado- que consista, como una melodía o una canción, esencialmente de sonidos. El inglés, en cambio, admite que se considere como Resultado causal de la acción del Actor cualquier objeto relacionable con el sonido (en principio, como soporte material o significante de ese objeto comunicativo). La Actividad 'whistle' en inglés puede referir así a un evento mucho más complejo y amplio que el denotado por el 'silbar' español. Por último, el ejemplo $(12 \mathrm{~g})$ representa una variante sutil de (12b); 'at+NP' -usada en sentido 'conativo' con verbos físicos como en 'cut at the bread' - presupone un grado de afectación menor que 'to+NP' en (12f). Podríamos captar en español este contraste apelando a la oposición acusativo/dativo ('le/lo') del mismo participante. En el caso de (12f) la afectación es mayor porque se trata de una orden mientras que en (12b) podría ser solo una expresión de agrado.

a. Kim whistled.

'Kim silbó'

b. Kim whistled at a pretty woman.

'Kim le silbó a la hermosa mujer'

c. Kim whistled a tune.

'Kim silbó una melodía’

d. Kim whistled a warning. 
Preferencia aspectual en el léxico y compensación morfo-sintáctica: Aspectos del contraste inglés-español / L. PARÍs

'Kim lanzó una advertencia mediante un silbido'

e. Kim whistled me a warning.

'Kim me lanzó una advertencia mediante un silbido'

f. Kim whistled to the dog to come.

'Kim lo silbó al perro para que viniera'

El español incluye menos verbos de Manera que el inglés, pero incluso aquellos que son parte de esta lengua, no pueden ser enriquecidos sintácticamente con un Resultado porque no se cuenta con una construcción - una asociación de forma sintáctica y contenido en el sentido de Construction Grammar (Goldberg, 2010)que lo permita.

\section{TRADUCCIÓN Y PARALELISMO INVERSO}

CGI y CR construyen representaciones de eventos según esquemas muy distintos. Es esperable, entonces, que sus traducciones sean desafiantes. Si bien es posible conllevar la misma información semántica, no es posible estructurarla del mismo modo y esto entraña una considerable diferencia comunicativa.
a. El niño entró a su dormitorio corriendo.

a'. The child ran into his bedroom.

Si bien estas oraciones contienen la misma información cognitivo-conceptual, su organización es diferente en dos aspectos fundamentales. Hemos visto ya que la Manera de movimiento y el Trayecto son introducidos en español en una frase adjunta -el gerundio- mientras que en inglés aparecen en el verbo principal. La diferencia insalvable está en la estructura pragmática. El gerundio en CGI constituye un componente imprescindible de la aseveración -por oposición a la presuposición-y por lo tanto, atrae al Foco pragmático. La información sobre Manera queda resaltada -la información 'nueva', impredecible es aportada por 'corriendo'- mientras que en la expresión inglesa Manera no tiene un rol pragmático sobresaliente. Podemos constatar esta afirmación con elementos sensitivos al Foco como la negación y los adverbios de frecuencia eventiva usados en (14) y (15), respectivamente. 
El niño no entró a su dormitorio corriendo.

El niño siempre entra a su dormitorio corriendo.

Enunciada (14) con una entonación típica, el adverbio de negación afecta al gerundio pero no al verbo principal y esto es posible solo si la cláusula principal es presupuesta y, por lo tanto, queda fuera del Foco. El niño entró pero no lo hizo corriendo. Por el contrario, el adverbio 'siempre' afecta la presuposición y de aquí que en (15) la cuantificación universal recae sobre los eventos de entrar al dormitorio. Todos ellos están incluidos en la afirmación, no así los eventos de correr; sólo un subconjunto de ellos, en particular, los asociados a entrar al dormitorio. Ambas pruebas arrojan la misma conclusión: en CGI la frase de gerundio es Foco mientras la cláusula principal presuposición. La consecuencia que nos interesa es que, entonces, Manera es Foco por defecto y esto explica que, como ya fue mostrado en el análisis del ejemplo (3), no sea posible expresarla si es predecible. En inglés la estructura de Foco es totalmente diferente. Manera aparece en un constituyente -el verbo principal- que no puede ser considerado Foco único en una interpretación asociada a la entonación no marcada. De tal modo que si bien la información puede ser la misma en inglés y español, el mensaje es distinto ya que el punto saliente en español es Manera mientras que en inglés es más bien el conjunto Manera, Trayecto y Meta.

El análisis de CR con los mismos parámetros muestra que la cláusula principal es parte de la presuposición mientras que la frase adjetiva FA atrae el Foco restringido.

John didn't shoot the sheriff dead.

Mary didn't wipe the table clean.

En (16) la interpretación no marcada es que John le disparó pero no mató al policía; en (17) sabemos que Mary repasó la mesa pero no quedó limpia. En ambos casos, el elemento saliente que se quiere comunicar es el Resultado. La traducción más literal al español no respeta esa estructura de información.

El policía no mató al ladrón de un tiro/al dispararle/disparándole.

(19) María no limpió la mesa repasándola.

El ejemplo (18) es un equivalente cognitivo de (16) pero comunicativamente presenta el Resultado como presuposición y no como Foco y lo mismo sucede entre (19) y (17). Por ejemplo, en (18) se dice que el policía mató al ladrón pero 
no de un tiro mientras que el inglés en (16) dice típicamente que le disparó pero no llegó a matarlo.

En síntesis, las equivalencias cognitivo-conceptuales de CGI en inglés así como la de $\mathrm{CR}$ en español presuponen transformaciones pragmáticas que alteran severamente el sentido de la comunicación. La explicación profunda de este contraste reside en la relación más general entre léxico y pragmática: lo que es saliente en el léxico -es decir, lo que está explícitamente codificado- tiende a perder relevancia cuando compite por el rasgo Foco con información léxicamente no especificada y expresada en la oración.

Este 'paralelismo inverso' tiene una complicidad sintáctica imprescindible y fundamental. Ambas construcciones, CGI y CR, se sustentarían en un mismo esquema sintáctico: ambas podrían ser analizadas como instancias de predicación secundaria. En una predicación secundaria, una cláusula principal domina otra cláusula 'defectiva' encabezada por un verboide -frase de gerundio- o un adjetivo -frase adjetiva. La noción de cláusula 'defectiva' remite al hecho de que, primero, carece de sujeto explícito y, segundo, tampoco tiene una interpretación temporal independiente (no contiene flexión temporal). En ambos casos la información la provee la cláusula principal.

Resulta fundamental para ponderar el contraste que intento dilucidar el hecho de que, en realidad, en español el esquema sintáctico que vertebra a CR en inglés existe y es, en realidad, muy frecuente. El español permite la Predicación Descriptiva ('dipictive predication') tal como se muestra en (20) y donde la frase subordinada es adjetiva (tal como CR).

$$
\text { El niño llegó a casa cansado. }
$$

Aquí 'cansado' es un adjetivo opcional -un adjunto sintáctico- que predica del sujeto del verbo principal ('el niño') una propiedad en relación al intervalo temporal especificado en la cláusula principal. El inglés puede replicar (21) con exactitud.

The child came home tired.

El esquema sintáctico que subyace a (20) y (21) es el mismo que corresponde a CR. La conclusión es clara, la única razón por la que el español no cuenta con una versión de CR tiene que ser pura y exclusivamente semántica. Esta perspectiva es exactamente opuesta a la asumida en Mateu (2012) sobre el mismo problema. Ese artículo resume otros del mismo autor en defensa de la tesis que CR en inglés es posible gracias al proceso 'conflation' que es diametralmente distinto al de 'incorporation', éste subyace a CGI en español. Sin entrar en detalle -que, por otro lado, inundan este tema- mi perspectiva semántica se ve, a mi juicio, irrefutablemente 
beneficiada frente a la sintáctica por la presencia de los mismos patrones sintácticos en ambas lenguas. Es más, como es esperable de fenómenos semánticos, existen ejemplos resultativos en español ligados a elementos léxicos ('Juan pintó la casa blanca'), es decir, carentes de toda productividad. Esta singularidad léxica no es esperable de un patrón sintáctico que, por definición, es abstracto y, por lo tanto, aplicable a un conjunto -típicamente numeroso- de elementos léxicos.

\section{LA REPRESENTACIÓN DE EVENTOS EN LA DIÁTESIS CAUSATIVA}

La morfosintaxis de la construcción de representaciones de eventos en español e inglés tiene otra diferencia fundamental relacionable con el procedimiento usado en las respectivas construcciones compensatorias arriba descritas. El español muestra una tendencia clara a codificar eventos complejos en el significado verbal, es decir, eventos que contienen subeventos gramaticalizados. El caso típico es el de los verbos causativos de cambio de estado como 'derretir', 'congelar', 'podrir', 'cocinar', 'descomponer', 'hundir', 'agrandar', 'achicar', etc. y verbos psicológicos como 'molestar', 'enojar', 'preocupar', 'asustar', 'disgustar', 'aburrir', etc.

$$
\text { El frío congeló el agua del balde. }
$$

El submarino hundió un barco.

'Congelar' en (22) refiere un evento causativo y, por lo tanto, se trata de una estructura bi-eventiva donde el primer subevento involucra un Agente (el frío) que causa un cambio de estado en un Paciente (el agua). Se trata de un Evento, en particular, una Realización puesto que es posible decir 'El frío congeló el agua en una hora'; esto implica, además, que 'el agua estuvo congelándose durante toda esa hora'. 'Hundir' es un evento causativo que entraña un Agente que hace algo y esta acción inespecífica causa un cambio de lugar y, por lo tanto, de estado en un Paciente ('el barco'). Se trata también de una Realización causativa ya que el verbo implica un cambio de estado final al término de un proceso durativo ('El submarino hundió un barco en dos minutos'). En español el morfema 'se' es aplicado a ambos verbos y tiene como efecto semántico el recorte de una representación bi-eventiva y causal que queda reducida a un solo evento, el subevento consistente en un cambio de estado.

(24) El agua se congeló.

(25) Un barco se hundió. 
La oración en (24) denota un Evento, en particular una Realización, que tiene como término un cambio de estado sufrido por un Paciente (el agua) luego de un proceso durativo ('el agua se congeló en diez minutos' entraña que estuvo diez minutos congelándose. Por su parte, (25) denota un Evento donde un Paciente cambia de estado luego de un proceso ('un barco se hundió en cinco minutos') previo a alcanzar ese punto télico de culminación.

El español parte de un verbo con una morfología simple (raíz verbal+flexión temporal) que denota una semántica compleja -en particular, un Evento complejo constituido de dos subeventos causalmente relacionados- y luego mediante reglas léxicas se accede a una morfología más compleja (raíz verbal+se+flexión temporal) que denota un evento más simple, es decir, un único subevento. En síntesis, la forma más simple en español se asocia con la semántica más compleja a la que descompone mediante una derivación morfológica. El inglés sigue un camino opuesto como lo muestra el contraste entre (26a) y (26b) con (27a) y (28b), respectivamente.

a. The water in the bucket froze.

b. The cold weather froze the water in the bucket.

a. The ship sank.

b. The submarine sank the ship.

La forma morfológicamente más simple es raíz+flexión temporal y denota la semántica más simple, es decir, la que consiste en un único evento de cambio de estado como en (26a) y (27a). La semántica más compleja (bi-eventiva y causal) es denotada mediante un proceso de transitivización de los verbos en (26b) y (27b), es decir, un proceso sintáctico que constituye una forma más compleja.

El léxico inglés parte de una semántica simple que se complejiza por medios sintácticos. La construcción de representaciones de eventos es composicional. El español, por el contrario, es des-composicional o substractiva, el léxico nos entrega un todo complejo que la derivación morfológica descompone en formas más simples. La construcción resultativa del inglés puede interpretarse como la composición de un evento mayor mediante la sintaxis a partir de una Actividad. Existe un paralelismo entre esta adición de material semántico mediante procedimientos sintácticos y el procedimiento -ya descrito- subyacente a CR. Inversamente, CGI en español consiste en la explicitación de una Actividad implícitamente ya contenida en el todo, una parte del evento ya introducido por el verbo principal. Es decir, el verbo principal (por ejemplo, 'entrar') alude a todo el evento referido en (2) mientras que el gerundio 'corriendo' especifica una parte de ese evento, no suma 
un (sub)evento. El español, tanto en SGI como en la diátesis causativa muestra que el punto de partida léxico es un todo semántico que luego descompone con la ayuda de procedimientos morfosintácticos.

Finalmente, es relevante señalar la sencillez con que el inglés deriva verbos de nombres, es decir, la aparente simpleza formal para generar a partir de un objeto a una estructura temporal dinámica. Esto es particularmente notorio con nombres que designan objetos que devienen integrados a una estructura causal bi-eventiva, donde un Agente causa que el objeto en cuestión se mueva -y, por lo tanto, funcione como Tema- hacia una Meta.

You have to stamp that envelop.

Mary wallpapered her bedroom.

(30) I buttered my toast.

En los tres casos un objeto -estampilla, papel, manteca- es un Tema que termina localizado en una Meta a causa de una acción dinámica ejecutada por un Agente. Se trata una especificación del esquema semántico abstracto que corresponde al verbo 'poner' (un Agente causa al ejecutar una acción que un Tema cambie de lugar para localizarse en una Meta). De hecho, los equivalentes en español típicamente son perífrasis con el verbo 'poner' ('poner una estampilla'; 'poner (o untar) manteca en el pan') o una morfología derivativa con el prefijo causativo 'en' y la terminación verbal ('empapelar'). El contraste apunta a una propiedad formal. En el caso de la construcción de eventos centrados en un objeto situado en el medio del desarrollo temporal de un evento, el inglés facilita notoriamente el paso de nombre (del objeto central) a verbo. Esta 'derivación cero' también es posible a partir del objeto Meta (en vez de Tema).

(31) The cowboy corralled the horses yesterday.

(32) The clerk boxed my toys before I could say anything.

(33) The police jailed the burglars.

(34) The hunter trapped the fox we had been looking for.

En síntesis, la integración de un objeto a un evento, es decir, la creación de una representación temporal dinámica a partir de un objeto, es un procedimiento facilitado por la morfología inglesa. Por el contrario, ese procedimiento en español no 
es productivo, el procedimiento consiste en añadir un afijo derivativo al nombre o producir una perífrasis para lograr el mismo efecto. ¿Qué relevancia tienen estos datos para la hipótesis aspectual? Por cierto, es indirecta. La preferencia por las Actividades del inglés resalta el rol de las representaciones dinámicas y continuas frente al resaltado de límites que caracterizan a los Eventos, el tipo preferido del léxico español. El léxico español marca (en el sentido literal de asignar un morfema diferente) la diferencia entre la referencia a un objeto y un proceso dinámico. No es accidental tampoco que la derivación cero aparezca en español en la dirección inversa, es decir, en la derivación de nombres a partir de verbos. Sin necesidad de añadir morfemas, la forma infinitiva del verbo español puede operar como sujeto u objeto preposicional e, incluso, recibir artículo y modificadores adjetivales ('El sonoro correr del agua por el arroyo encantaba a los turistas').

\section{CONCLUSIÓN}

En este artículo se ha propuesto que el inglés y el español se caracterizan por patrones distintos en sus ensamblajes de sintaxis, semántica y pragmática para construir representaciones de eventos. La diferencia tiene un origen léxico-semántico. En una reinterpretación y generalización de la tipología de Talmy, se ha propuesto que el inglés privilegia en el léxico las Actividades mientras el español prefiere Eventos, tesis que denomino 'preferencia aspectual'. La evidencia en relación a los verbos de movimiento es contundente y ha sido en parte aquí revisitada. Se ha propuesto y mostrado, además, que estas lenguas balancean sus desequilibrios léxicos -hipótesis de la 'compensación'- mediante construcciones sintácticas. El español ofrece la Construcción de Gerundio Interno que permite enriquecer la descripción de un Evento mediante la especificación de una Actividad interna al mismo. El inglés, en cambio, equilibra el repertorio menguado de Eventos con la Construcción Resultativa que permite enriquecer una Actividad mediante el añadido de su Resultado, y se obtiene así un Evento. Es crucial para las tesis de la preferencia aspectual y de la compensación que la Construcción Resultativa no exista en español y más aún, que exista una construcción sintácticamente idéntica -la Construcción de Predicado Secundario-, porque se corrobora así que aquella ausencia está solo semánticamente motivada, precisamente lo que esperamos a partir de la tesis de la 'preferencia'. Es también esperable a partir de la tesis compensatoria que la Construcción de Gerundio Interno sea inmaterial en inglés y esto es precisamente lo que se ha mostrado, su lánguida presencia se restringe a un tipo de verbo principal. Es así que la traducción de SGI al inglés y de CR al español resulta una tarea compleja que exige representar no solo la misma información sino también el mismo mensaje. 
Por último, he presentado un bosquejo de análisis contrastivo de otros dos fenómenos gramaticales que son consistentes con ambas hipótesis. El primero refiere a la codificación de verbos causativos de cambio de estado como 'hundir/ sink'. En español la forma (morfosintácticamente) más simple -y, por ende, la que asumimos como base léxica- codifica al Evento complejo, bi-eventivo, que incluye un subevento causante y otro causado de cambio de estado. La morfosintaxis ofrece el morfema 'se' -en sentido estricto, el clítico (pseudo)reflexivizante- para generar un verbo intransitivo ('hundirse') con una semántica simple, la del subevento cambio de estado que aquí ya no es más causado sino potencialmente espontáneo o, al menos, inespecífico respecto de la causalidad. Por el contrario, el inglés parte de la forma más simple asociada a la semántica más simple y mono-eventiva para generar mediante la sintaxis un verbo transitivo asociado a la semántica más compleja. El segundo fenómeno refiere a la simplicidad formal (morfosintáctica) del inglés para construir representaciones causativas con agentes dinámicos a partir de objetos. Esta simplicidad contrasta con la necesidad de recurrir a morfemas derivativos en español para obtener un resultado similar.

Sin bien la evidencia presentada es suficiente para sostener las dos hipótesis presentadas como razonablemente plausibles, puede y debe ampliarse en relación al espectro de verbos analizados ${ }^{6}$. La pregunta es siempre si la validez de estas afirmaciones se restringe a un número de clases de verbos -no importa qué tan grande- o si se expande sin excepción a través del léxico (obviamente, exceptuamos de esta evaluación las clases de verbos que no contienen un miembro que denote una Actividad, como los verbos psicológicos, o aquellas que no contengan Estados). En definitiva, estas hipótesis no abarcan pero sugieren una perspectiva más general todavía sobre el estilo de codificación que caracteriza a cada lengua, perspectiva que define una hipótesis de trabajo para explorar en el futuro. Se trata de asumir que el léxico inglés se inclinaría hacia lo dinámico, es decir, la acción y, por ende, al participante Actor. Frente a ello, el léxico español sería más sensible al cambio, es decir, al Paciente, el participante afectado por un (cambio de) estado. Una lengua con mayor fertilidad para generar representaciones de eventos dinámicos y la otra más propensa y preparada para representar Estados.

\footnotetext{
${ }^{6}$ En relación a la temporalidad, Sonia Suárez Cepeda lleva a cabo un estudio experimental en el que intenta captar las implicancias cognitivas de operar alternativamente con las estructuras temporales que caracterizan al inglés, el español y el chino. El grupo de Análisis Contrastivo de la UNCuyo lleva a cabo un proyecto de investigación para analizar la preferencia aspectual en una amplia gama de clases de verbos usando datos de corpus.
} 
Preferencia aspectual en el léxico y compensación morfo-sintáctica: Aspectos del contraste inglés-español / L. PARís

\section{REFERENCIAS}

Beaver, John y Koontz-Garboden, Andrew. (2012). Manner and Result in the roots of verbal meanings. Linguistic Inquiry, 43, 331-369.

Bowerman, Melissa y Choi, Sandra. (2003). Space under construction: Languagespecific spatial categorization in first language acquisition. En D. Gentner y $S$. Goldin-Meadow (eds.), Language and mind (pp. 387-429). Cambridge, MA: MIT Press.

Dowty, David. (1991). Thematic proto-roles and argument selection. Language, $67(3), 547-619$.

Foley, William y Mike Olson. (1985). Clausehood and verb serialization. J. Nichols and A. Woodbyry (ed.), Grammar inside and outside the clause (pp. 1760). London: Cambridge University Press.

Gennari, Silvia; Sloman, Steven; Malt, Barbara y Fitch, Tecumseh. (2002). Motion Events in language and cognition. Cognition, 83, 49-79.

Gentner, Dedre y Melissa Bowerman. (2009). Why some spatial semantic categories are harder to learn than others: The typological prevalence hypothesis. En Jiansheng Guo, Elena Lieven, Nancy Budwig, Susan Ervin-Tripp, Keiko Nakamura y Şeyda Özçalışkan (eds.), Cognitive approaches to the psychology of language (pp. 465-481). New York: Psychology Press.

Goldberg, Adele. (2010). Verbs, constructions and semantic frames. En Edit Doron y Ivy Sichel (eds.), Syntax, Lexical Semantics and Event Structures (pp. 3958). Oxford: Oxford University Press.

Goldberg, Adele y Ray Jackendoff. (2004). The English Resultative as a Family of Constructions. Language, 80 (3), 532-568.

Levinson, Steven. (2000). Presumptive meanings. Cambridge, MA: MIT Press.

Malouf, Robert. (2000). Mixed categories in the Hierarchical Lexicon. Stanford, CA: CSLI Publications.

Mateu, Jaume. (2012). Conflation and incorporation processes in resultative constructions. En Violeta Demonte y Louise McNally (eds.), Telicity, change, and state (pp. 252-278). Oxford: Oxford University Press.

París, Luis. (2006a). Implicating and focusing on underspecified lexical information. En Klaus von Heusinger and Ken Turner (ed.), When semantics meets pragmatics (pp. 421-444). Oxford: Elsevier.

París, Luis. (2006b). La codificación gramatical de Eventos: Medio $_{\mathrm{E}}$. Signos, 39 (61), 259-262.

París, Luis. (2006c). Conceptos en forma: IncidentalE y MedioE en frases de gerundio. RASAL, 5, 47-71.

París, Luis. (2008). On Expanditures. En Nikki Adams, Adam Cooper, Fey Parrill y Thomas Wier, Proceedings of the $40^{\text {th }}$ Regional Meeting of the Chicago Linguistic Society (pp. 291-304). Chicago: CLS. 
París, Luis. (2010) El significado como concepto estructurado. En Gisella Müller, Vanina Barbeito y Laura Miñones (eds.) Estudios de Lingüistica Cognitiva (pp. 241-257). Mendoza: EDIUNC.

París, Luis. (2011). Invisibilidad semántica de lo conceptual. En Virginia Jaichenco y Yamila Sevilla (eds.) Primer Encuentro de Procesamiento del Lenguaje (pp. 192-201). Buenos Aires: Editorial de la FFyL, UBA. Disponible en http:// il.institutos.filo.uba.ar/sites/il.institutos.filo.uba.ar/files/PROLEN\%202012. pdf

París, Luis. (2013). Adjuncts and the syntax-semantics interface: Spanish gerund constructions. En XIII Role and Reference Grammar International Conference, Friburgo, Alemania.

París, Luis y Jean Pierre Koenig. (2003). 'What does it mean to be a dependent?'. En Proceedings of the $10^{\text {th }}$ International Conference on Head Driven Phrase Structure Grammar (pp. 298-317). Stanford: CSLI.

Rappaport Hovav, Malka y Beth Levin. (2010). Reflections on Manner/Result complementarity. En E. Doron, M. Rappaport Hovav y I. Sichel (ed.), Syntax, lexical semantics, and Event structure (pp. 21-39). Oxford: Oxford University Press.

Real Academia Española. Nueva gramática de la Lengua Española. (2011). Madrid: Espasa.

Rothstein, Susan. (2000). Secondary predication and aspectual structure. En Fabricius-Hansen, Catherine, Ewald Lang y Claudia Maienborn (eds.), Approaching the grammar of adjuncts: Proceedings of the Oslo Conference. Disponible en http://www.zas.gwz-berlin.de/fileadmin/material/ZASPiL_Volltexte/ zp17/zaspil17-rothstein.pdf.

Slobin, Dan. (2000). Verbalized Events: A dynamic approach to linguistic relativity and determinism. In S. Niemeier y R. Dirven (eds.), Evidence for linguistic relativity (pp. 107-138). Amsterdam/Philadelphia: John Benjamins.

Slobin, Dan. (2006). What makes manner of motion salient? Explorations in linguistic typology, discourse, and cognition. En M. Hickmann y S. Robert (eds.), Space in languages: Linguistic systems and cognitive categories (pp. 59-81). Amsterdam: John Benjamins.

Talmy, Leonard. (2000). Toward a cognitive semantics. Cambridge, MA: MIT Press.

Talmy, Leonard. (2009). Main verb properties and equipollent framing. En Jiansheng Guo, Elena Lieven, Nancy Budwig, Susan Ervin-Tripp, Keiko Nakamura y Şeyda Özçalışkan (eds.), Cognitive approaches to the psychology of language (pp. 389-403). New York: Psychology Press.

Tenny, Carol. (2000). Core Events and adverbial modification. En Carol Tenny y James Pustejovsky (ed.), Events as grammatical objects. Dordrecht, Holanda: Kluwer. 
Preferencia aspectual en el léxico y compensación morfo-sintáctica: Aspectos del contraste inglés-español / L. PARís

Truswell, Robert. (2007). Extraction from adjuncts and the structure of Events. Lingua, 117, 1355-1377.

Van Valin, Robert D. (1990). Semantic parameters of split intransitivity. Language, 66, 221-60.

Van Valin, Robert D. (2005). Exploring the syntax-semantics interface. Cambridge: CUP.

Vendler, Zeno. (1957). Verbs and times. Philosophical Review, 66, 143-160. 